\title{
Simulation of a Two-Phase Microcapsule Spraying onto a Solid Wall
}

\author{
Zhimin Xie ${ }^{\mathrm{a}}$, Zhuwang Huang ${ }^{\mathrm{b}}$ and Yuyan Liu ${ }^{\mathrm{c}}$
}

National Key Laboratory of Science and Technology on Advanced Composites in Special

Environment, Center for Composite Materials, Harbin Institute of Technology, Harbin 150001, P.R.

China

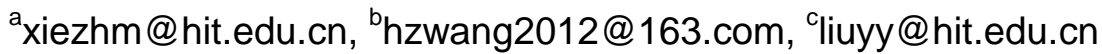

Keywords: Microcapsule; Spray; Critical velocity; Breakup; Impact.

Abstract: Spraying microcapsules with polymeric shell onto a solid wall may be a way to fabricate multilayer films. Better understanding of the deformation and breakup of the microcapsule under spraying is of great importance for developing this technique. A two-phase finite element model was developed using the software Autodyn to simulate the normal spraying process. In light of the dichotomy method, we obtained the critical velocity for a microcapsule to be broken. The simulations for various microcapsule shell thickness showed that the critical velocity increased with an increase of the shell thickness. The thermal analysis revealed that the maximum temperature of the microcapsule appeared around the center of the contact region at the beginning of the spraying, and later it moved to the edges of the contact region. The increased temperature of the microcapsule was $10.6 \mathrm{~K} \mathrm{at} 100 \mathrm{~m} / \mathrm{s}$, while it was amazing up to $180.5 \mathrm{~K}$ at $1000 \mathrm{~m} / \mathrm{s}$. High temperature might affect the film formation and then production quality after the release of the fluid.

\section{Introduction}

Microcapsules, commonly composed of a solid shell and an internal phase, find applications in the paper coating, medicine, self-healing material and many other fields. The stability of the microcapsules is related to the properties of the capsule shell. It has been known that ethyl cellulose, polyvinyl alcohol, gelatin and sodium alginate are generally used as the shell materials. Based on the mechanism of shell formation, the encapsulation techniques have been classified as interfacial, in situ, coacervation, meltable dispersion, or physical [1]. Recently, Gormley et al. have reported a special layer-by-layer assembly technique to obtain the stable hollow capsules [2]. Besides the shell materials, the shell thickness also affects the stability of the microcapsules. Once the tiny capsule is broken, the containing material, e.g. an adhesive or a medicine, will be released. The pressure, impact and temperature can trigger a rupture of the microcapsule shell. If the shell is made of a polymeric film and the released material can form a continuous film, it is anticipated that, spraying microcapsules with polymeric shell onto a solid wall could be used to fabricate multilayer films. Naturally, the final products are related to the impact conditions. The microcapsule cannot rupture until the spraying velocity is high enough; thus, determination of the critical spraying velocity is of great importance for developing this technique.

The impingement process depends on several factors such as, spraying velocity, shell thickness and size of microcapsule, material properties [3]. Experimental investigation can provide the relation between the processing parameters and the microcapsule information, but it will cost too much. In practice, the numerical simulations are popular methods to probe scientific insight to the impingement process. Computational fluid dynamics (CFD) based volume of fluid method (VOF) is widely utilized in tracking the deforming and spreading characteristics of the droplets. Based on a fixed Eularian structured mesh, Tabbara and $\mathrm{Gu}$ investigated the splat formation of a molten tin droplet impacting on a flat substrate using the VOF method to simulate the boundary between the metallic and atmospheric-gas phases [3]. They suggested that the solidification process consisted of the planar solidification, uneven solidification and wave mixing stages. Many efforts focused on the impact dynamics of dense melt droplets. Recently, the numerical results have indicated that the impingement process of hollow droplet with a liquid shell is distinctly different from the dense 
droplet, and the droplet void fraction and void distribution have a significant influence on the flow dynamics [4]. For a two-phase microcapsule, the CFD-based models are difficult to describe the process of deformation and breakup due to the presence of the elastic shell which leads to the different mechanical behaviors in comparison with the liquid droplet. Since the explicit finite element (FE) method has been successfully applied to the simulation of projectile dynamic impact $[5,6]$, in the present work, we simulated the spraying process of an microcapsule using a two-phase FE model, where the solid shell and the internal liquid of the microcapsule were modeled using the methods of Arbitrary Lagrange-Euler (ALE) and Smoothed Particle Hydrodynamics (SPH), respectively. The critical conditions for the microcapsule to be broken under spraying were obtained; variations of the temperature of the microcapsule-wall system with the spraying velocity were further studied.

\section{Numerically computational model}

Consider a spherical microcapsule with inner diameter of $280 \mu \mathrm{m}$ and shell thickness of $10-30 \mu \mathrm{m}$ spraying onto a flat solid wall with thickness of $200 \mu \mathrm{m}$. The microcapsule was assumed to consist of the polyurethane (PU) shell and filled water; the solid wall was assumed to be made of aluminum (Al). It should be stated that the following simulation method for these materials is also applicable to the other materials. For simplicity, the microcapsule under normal spraying was modeled using the commercial FE software Autodyn as a 2D two-phase structure, where the solid shell and filled liquid were described using the methods of ALE and SPH, respectively. The Lagrange method was utilized to the $\mathrm{Al}$ wall, for the target wall had a small deformation under spraying. A boundary condition of fixed support was applied to one edge of the Al wall. In view of the structural symmetry, the numerical model was computed for half a microcapsule, as shown in Fig. 1.

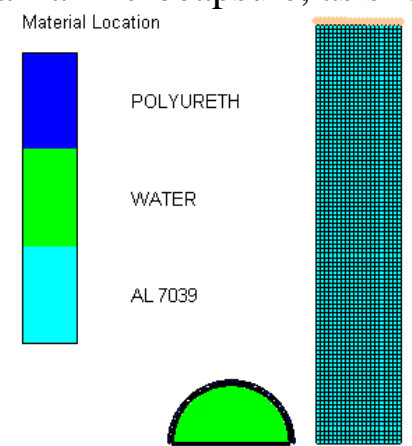

Fig. 1. Numerical computational model for a microcapsule spraying onto a solid wall

The material parameters for PU, water and Al were listed in Table. 1 along with the Shock equation of state (EOS) provided by the Autodyn. The Al wall was represented using a Johnson-Cook strength model. The mechanical behaviors of the PU shell were described by a viscoelastic model in terms of stress deviators $\sigma_{i j}^{\prime}$, i.e.,

$$
\sigma_{i j}^{\prime}=2 \int_{0}^{t} \phi(t-\tau)\left[\frac{\partial \varepsilon_{i j}^{\prime}(\tau)}{\partial t}\right] d \tau \text { and } \phi(t)=G_{\infty}+\left(G_{0}-G_{\infty}\right) e^{-\beta t},
$$

where $\mathrm{G}_{\infty}=22.4 \mathrm{MPa}, \mathrm{G}_{0}=29.9 \mathrm{MPa}$ and $\beta=1.35$. The breakup of the microcapsule was determined according to the maximum tensile stress failure criterion; here, the ultimate failure stress was set to 34.5MP.

Table 1 The material parameters

\begin{tabular}{ccccc}
\hline Material & $\rho_{0}\left(\mathrm{~kg} / \mathrm{m}^{3}\right)$ & $\mathrm{C}_{1}(\mathrm{~m} / \mathrm{s})$ & $\mathrm{S}_{1}$ & $\Gamma_{0}$ \\
\hline Water & 998 & 1647 & 1.921 & 0 \\
$\mathrm{PU}$ & 1265 & 2486 & 1.577 & 1.55 \\
$\mathrm{Al}$ & 2770 & 5328 & 1.338 & 2 \\
\hline
\end{tabular}




\section{Results and discussion}

The simulation calculations were conducted with the software Autodyn. The spraying process of a microcapsule onto an $\mathrm{Al}$ wall at $39 \mathrm{~m} / \mathrm{s}$ is shown in Fig. 2. The microcapsule deformed largely at the beginning of spraying, followed by breakup and release of fluid. At the failure region of the shell, a Gauss point was selected in the simulation to track the evolution of stress. As can be seen in Fig. 3, the stress at the Gauss point increased gradually with time. At $2.80 \mu \mathrm{s}$, the stress reached a maximum $34.5 \mathrm{MPa}$, up to the ultimate failure stress; then the microcapsule began to break and the stress dropped sharply to zero. Fig. 2 (d) also shows the release of the filled fluid at $4.03 \mu \mathrm{s}$.

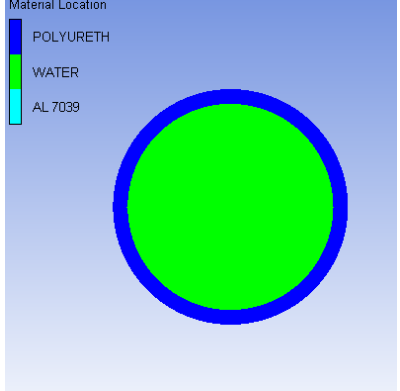

(a)

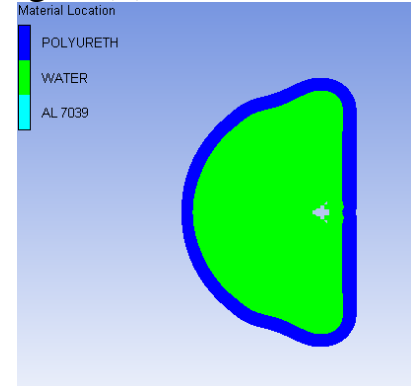

(b)

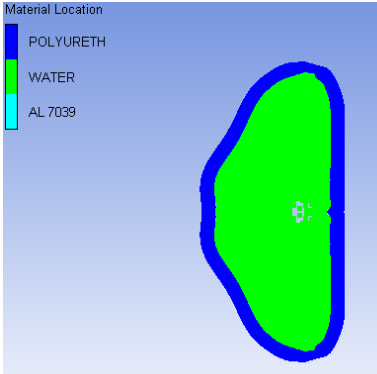

(c)
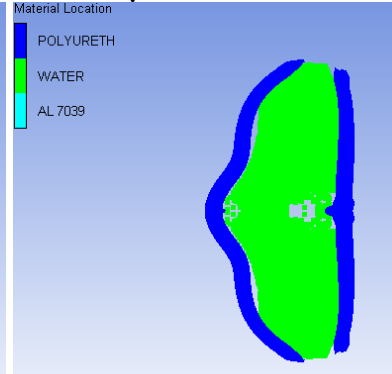

(d)

Fig. 2. The deformation of a microcapsule at (a) $0 \mathrm{~s}$, (b) $2.06 \mu \mathrm{s}$, (c) $3.37 \mu \mathrm{s}$, (d) $4.03 \mu \mathrm{s}$.

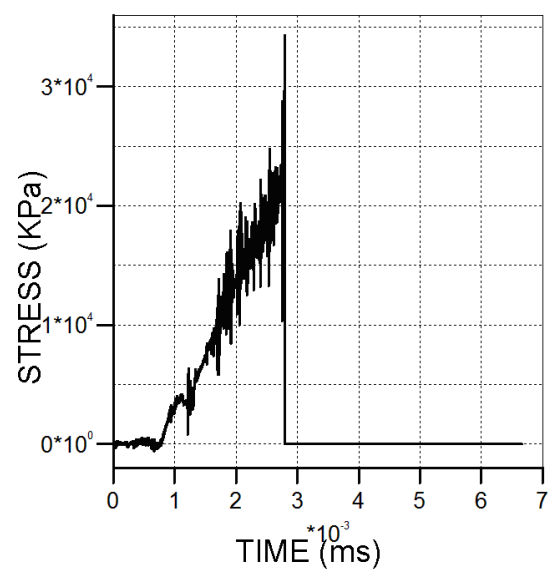

Fig. 3. The stress at a Gauss point.

When the spraying velocity is lower than the threshold value at which the rupture occurs, the microcapsule will be rebounded. It is difficult to obtain a formulated threshold. In light of the dichotomy method, we obtained a narrow range in which the critical value lay by several calculation times. Hence, the critical velocity can be evaluated numerically. For a given precision $\varepsilon$, the minimum calculation times $(n)$ can be estimated as $n=\log _{2} \frac{V 2-V 1}{\varepsilon}$ at the velocity range of [V1,V2]. Clearly, the microcapsule remained intact at the spraying velocity of zero. A trial calculation was performed at $1000 \mathrm{~m} / \mathrm{s}$; the microcapsule was found breakup. Thus, set the calculating precision $\varepsilon=2 \mathrm{~m} / \mathrm{s}$ and the spraying velocity rang from 0 to $1000 \mathrm{~m} / \mathrm{s}$. We got the critical velocity $38.08 \mathrm{~m} / \mathrm{s}$ for the shell thickness of $20 \mu \mathrm{m}$ through nine times simulations.

To study the effect of the shell thickness on the critical velocity, the simulations were performed for the spraying microcapsules with shell thickness of $10 \mu \mathrm{m}, 15 \mu \mathrm{m}, 20 \mu \mathrm{m}, 25 \mu \mathrm{m}$, and $30 \mu \mathrm{m}$. Fig. 4 shows the relation between the critical velocity and the shell thickness. It was found that the critical velocity increased with an increase of the shell thickness. From the relation curve in Fig. 4, one can easily reach the critical spraying velocity for a given shell thickness, or the shell thickness to be broken for a spraying velocity. 


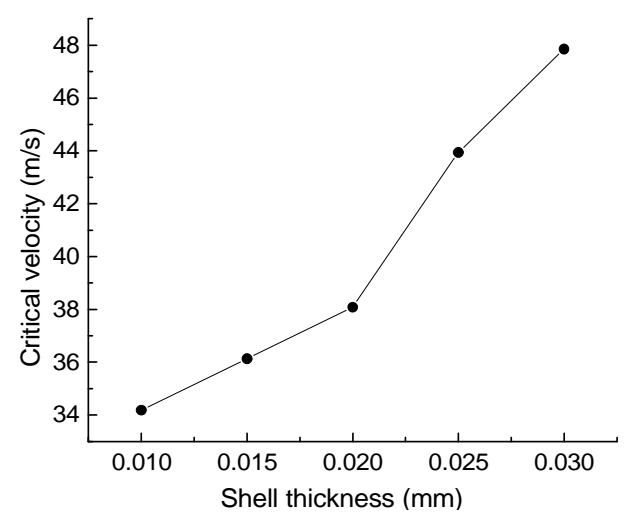

Fig. 4. Relation between the critical velocity and the shell thickness.

From the energy point of view, the kinetic energy of the microcapsule was converted to the deformation energy and intrinsic energy of the microcapsule-wall system. The latter energy leaded to an increase in temperature of the system. High temperature might affect the film formation and then production quality after the release of the fluid. Therefore, we focused on the temperature changes of the microcapsule and $\mathrm{Al}$ wall during spraying at $100 \mathrm{~m} / \mathrm{s}$. The initial temperature of the microcapsule-wall system was set to $293 \mathrm{~K}$. The temperature distributions calculated for the shell $(10 \mu \mathrm{m}$ in thickness) and the filled water were plotted in Fig. 5 and Fig. 6, respectively. Fig. 7 shows the surface temperature of the $\mathrm{Al}$ wall at the spraying point, revealing little change in temperature. At the beginning of the spraying, the maximum temperature of the microcapsule appeared around the center of the contact region; later it moved to the edges of the contact region. The microcapsule gained a maximum $10.6 \mathrm{~K}$ increase in temperature around the contact region. When the spraying velocity was $1000 \mathrm{~m} / \mathrm{s}$, the increased temperature was amazing up to $180.5 \mathrm{~K}$. Fig. 8 shows the variation of the maximum pressure acting on the flat wall with the spraying velocity. As expected, the high spraying velocity resulted in the high pressure. If the wall thickness was thin enough, the local deformation and failure of the wall should be taken into account.

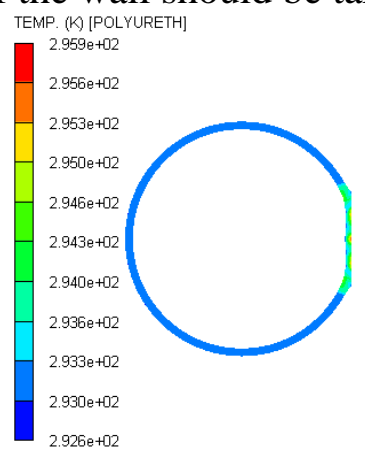

(a)

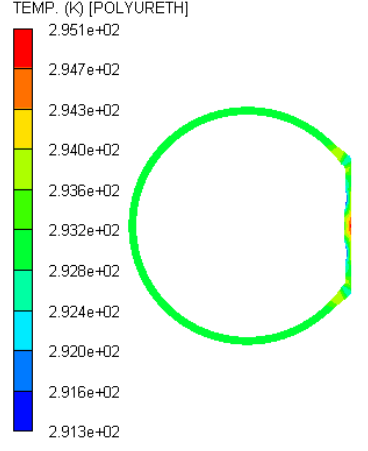

(b)

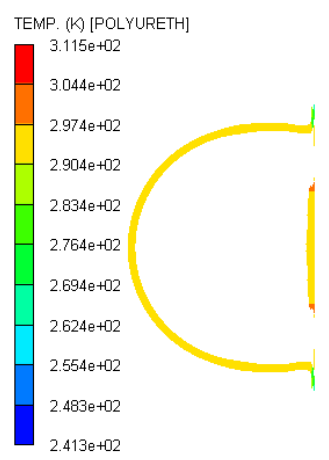

(c)

Fig. 5. The temperature of the shell at (a) $0.09 \mu \mathrm{s}$, (b) $0.19 \mu \mathrm{s}$, (c) $0.64 \mu \mathrm{s}$.

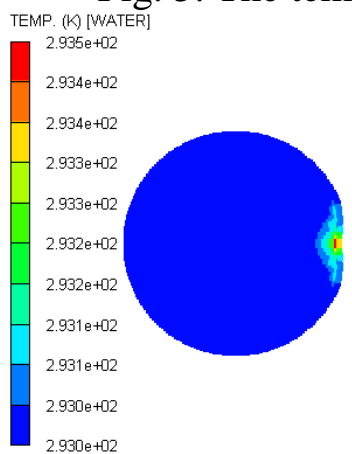

(a)

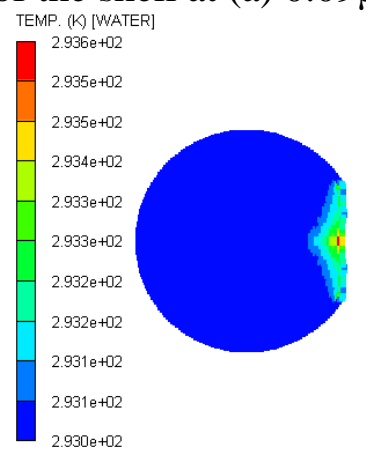

(b)
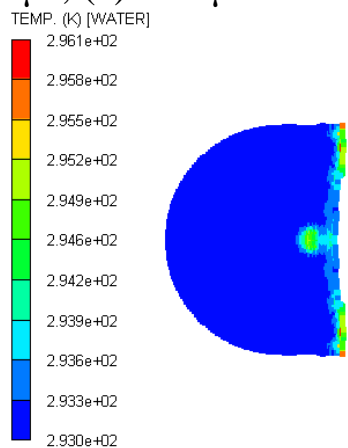

(c)

Fig. 6. The temperature of the water at (a) $0.09 \mu \mathrm{s}$, (b) $0.19 \mu \mathrm{s}$, (c) $0.64 \mu \mathrm{s}$. 


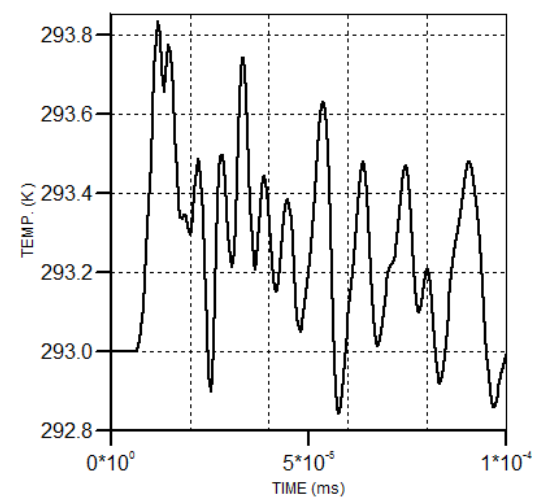

Fig. 7. The surface temperature of the $\mathrm{Al}$ wall at the spraying point.

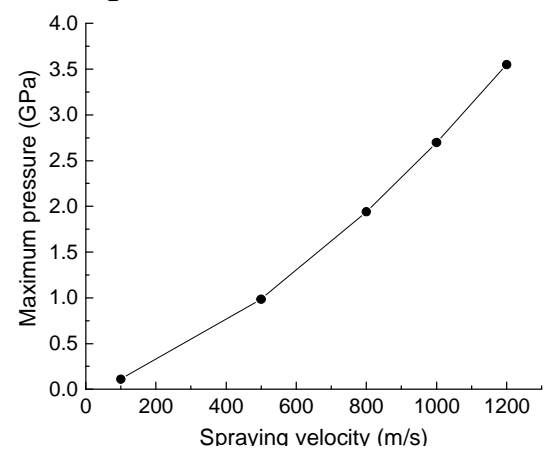

Fig. 8. The variation of the maximum pressure with the spraying velocity.

\section{Conclusions}

A two-phase finite element model was developed using the software Autodyn to simulate the microcapsule spraying process. It was found that the critical velocity for a microcapsule to be broken can be easily determined by means of the dichotomy. The simulations for various microcapsule shell thickness of $10 \mu \mathrm{m}, 15 \mu \mathrm{m}, 20 \mu \mathrm{m}, 25 \mu \mathrm{m}$, and $30 \mu \mathrm{m}$ showed that the critical velocity increased with an increase of the shell thickness. The thermal analysis revealed that the maximum temperature of the microcapsule appeared around the center of the contact region at the beginning of the spraying, and later it moved to the edges of the contact region. The increased temperature of the microcapsule was $10.6 \mathrm{~K}$ at $100 \mathrm{~m} / \mathrm{s}$, while it was amazing up to $180.5 \mathrm{~K}$ at $1000 \mathrm{~m} / \mathrm{s}$. For the $\mathrm{Al}$ wall, there was little change in temperature at the spraying point. Additionally, calculations also revealed that the high spraying velocity resulted in the high pressure acting on the flat wall. If the wall thickness was thin enough, its deformation and failure should be taken into account.

\section{References}

[1] B.J. Blaiszik, S.L.B. Kramer, S.C. Olugebefola et al.: Annu. Rev. Mater. Res. 40 (2010) 179-211.

[2] A.J. Gormley, R. Chandrawati, A.J. Christofferson et al.: Chem. Mater. 27 (2015) 5820-5824.

[3] H. Tabbara, S. Gu: Int. J. Heat Mass Transfer. 55 (2012) 2081-2086.

[4] A. Kumar, S. Gu: Int. J. Heat Fluid Flow. 37 (2012) 189-195.

[5] T. Børvik, M. Langseth, O.S. Hopperstad, K.A. Malo: Int. J. Impact Eng. 22 (1999) 855-886.

[6] A.V. Potapov, M.L. Hunt, C.S. Campbell: Powder Technol. 116 (2001) 204-213. 